\title{
Clinical significance of neutrophil-to- lymphocyte ratio as a predictor of lymph node metastasis in gastric cancer
}

Toshiyuki Kosuga* (D), Tomoki Konishi, Takeshi Kubota, Katsutoshi Shoda, Hirotaka Konishi, Atsushi Shiozaki, Kazuma Okamoto, Hitoshi Fujiwara, Michihiro Kudou, Tomohiro Arita, Ryo Morimura, Yasutoshi Murayama, Yoshiaki Kuriu, Hisashi Ikoma, Masayoshi Nakanishi and Eigo Otsuji

\begin{abstract}
Background: Precise staging is indispensable to select the appropriate treatment strategy for gastric cancer (GC); however, the diagnostic accuracy of conventional modalities needs to be improved. This study investigated the clinical significance of the preoperative neutrophil-to-lymphocyte ratio (NLR) for the prediction of pathological lymph node metastasis ( $\mathrm{pN}+$ ) in GC.

Methods: This was a retrospective study of 429 patients with GC who underwent curative gastrectomy. The predictive ability of NLR for $\mathrm{pN}+$ was examined in comparison with that of computed tomography.

Results: The preoperative NLR ranged from 0.6 to 10.8 (median, 2.0), and the optimal cut-off value for predicting pN+ was 1.6 according to the receiver operating characteristic curve with the maximal Youden index. Multivariate analysis identified a NLR $\geq 1.6$ (odds ratio (OR) 3.171; 95\% confidence interval (Cl) 1.448-7.235, $p=0.004$ ) and cN+ (OR 2.426; 95\% Cl 1.221-4.958, $p=0.011$ ) to be independent factors associated with pN+ in advanced GC (cT2-T4). On the other hand, a $\mathrm{NLR} \geq 1.6$ was not useful for predicting pN+ in early GC (CT1). In advanced GC, a NLR $\geq 1.6$ detected pN+ with a higher sensitivity (84.9\%) and negative predictive value (NPV) (63.9\%) than conventional modalities (50.0 and $51.7 \%$, respectively). When the subjects were limited to those with advanced GC with cNO, the sensitivity and NPV of a NLR $\geq 1.6$ for pN+ increased further ( 90.7 and $81.0 \%$, respectively).
\end{abstract}

Conclusion: The preoperative NLR may be a useful complementary diagnostic tool for predicting pN+ in advanced GC because of its higher sensitivity and NPV than conventional modalities.

Keywords: Gastric cancer, Gastrectomy, Neutrophil-to-lymphocyte ratio, Lymph node metastasis, Staging

\section{Background}

Gastric cancer (GC) is the fifth most common cancer and the third leading cause of cancer-related death worldwide [1]. Gastrectomy with prophylactic lymphadenectomy is the basic surgical concept for GC, and treatment outcomes have been improving with advances in surgical techniques $[2,3]$. In patients with early GC (EGC), D1/D1+ or D2 is adopted for those with cN0 or $\mathrm{cN}+$, respectively [4]. On the other hand, in patients with advanced GC (AGC), D2 has been uniformly performed regardless of $\mathrm{cN}$ because the incidence of $\mathrm{pN}+$ is

* Correspondence: toti-k@koto.kpu-m.ac.jp

Division of Digestive Surgery, Department of Surgery, Kyoto Prefectural

University of Medicine, 465 Kajii-cho, Kamigyo-ku, Kyoto 602-8566, Japan high but difficult to precisely predict [4]. Preoperative assessment of lymph node metastasis is generally performed by computed tomography $(\mathrm{CT})$ [5]; however, the diagnostic accuracy for $\mathrm{pN}+$ is not sufficient and should be improved using another diagnostic tool.

Cancer-related inflammation was previously confirmed as a key determinant of cancer progression, and systemic inflammation is associated with alterations in peripheral blood leukocytes that are reflected in the neutrophil-tolymphocyte ratio (NLR) [6-8]. Therefore, NLR may be a complementary diagnostic tool for $\mathrm{pN}+$. There are many reports demonstrating the prognostic impact of NLR in different cancers [9-11]; however, few studies have

(c) The Author(s). 2019 Open Access This article is distributed under the terms of the Creative Commons Attribution 4.0 International License (http://creativecommons.org/licenses/by/4.0/), which permits unrestricted use, distribution, and 
examined whether NLR is useful for predicting $\mathrm{pN}+$ in comparison with conventional diagnostic modalities.

In the present study, the diagnostic accuracy of NLR for $\mathrm{pN}+$ was compared with that of conventional modalities such as CT. The aim of this study was to explore the clinical significance of NLR as a predictor of $\mathrm{pN}+$ in GC, and to examine whether NLR can improve the diagnostic accuracy for $\mathrm{pN}+$ in combination with conventional modalities.

\section{Methods}

\section{Patients}

Between January 2008 and May 2013, 578 patients underwent surgical treatment for GC at the Division of Digestive Surgery of Kyoto Prefectural University of Medicine (KPUM). Among these patients, this study examined only those who underwent CT followed by curative gastrectomy (R0). The following exclusion criteria were applied to potential subjects of this study: active infection, chronic inflammatory or autoimmune diseases, chronic use of steroid and/or immunosuppressive agents, hematological disorders, lack of information on preoperative complete blood counts, distant metastasis of GC, and simultaneous malignancies other than GC. In addition, to exclude the potential effects of treatment factors on the diagnostic accuracy for $\mathrm{pN}+$, patients who underwent non-curative surgery (R1/R2) and those undergoing neoadjuvant chemotherapy (NAC) were also excluded. In contrast to Western countries, NAC has not been the standard treatment for GC even with advanced stage in Japan [4]. In total, 429 patients were included in this retrospective study.

\section{Assessment of NLR}

The preoperative cell blood count $(\mathrm{CBC})$ and differential white blood cell count (WBC), including neutrophils and lymphocytes, were measured within one week before surgery. NLR was calculated as the absolute neutrophil count divided by the absolute lymphocyte count.

\section{Assessment of $\mathrm{cT}$ and $\mathrm{cN}$}

All patients received upper endoscopy, barium meal examination, and chest and abdominal CT prior to surgery. The clinical $\mathrm{T}$ stage (cT) was assessed using upper endoscopy, barium meal examination, and abdominal CT findings by gastroenterologists and radiologists. The clinical $\mathrm{N}$ stage $(\mathrm{cN})$ was diagnosed using the chest and abdominal CT findings by at least two radiologists. CT was performed at KPUM or Oike Clinic (Kyoto, Japan), a consociated medical center, employing a multidetector CT with 64 or 320 layers. Contrast-enhanced CT (CECT) with iopamidol or iohexol was the recommended standard, but patients who had iodine allergy, active asthma, or poor thyroid, heart, liver or renal function underwent plain CT without the contrast agents. Lymph nodes exhibiting a minor axis of $8 \mathrm{~mm}$ or greater or a major axis of 10 $\mathrm{mm}$ or greater on $\mathrm{CT}$ were regarded as " $\mathrm{CN}+$ " according to previous studies $[5,12,13]$. Endoscopic ultrasound (EUS) was not used for the evaluation of $\mathrm{cN}$.

\section{Assessment of $\mathrm{pT}$ and $\mathrm{pN}$}

All patients underwent R0 surgery consisting of gastrectomy and lymphadenectomy based on the Japanese GC treatment guidelines (JGCTG) [4]. All resected specimens were microscopically examined by at least two pathologists, and the pathological $\mathrm{T}$ and $\mathrm{N}$ stages (pT and $\mathrm{pN}$ ) were evaluated based on the current Japanese classification of GC (JCGC) [14].

\section{Statistical analysis}

To evaluate the discriminatory ability of the NLR for $\mathrm{pN}+$, receiver operating characteristic (ROC) curves were generated and the area under the ROC curve (AUROC) was measured. The optimal cut-off value of NLR was determined by the Youden index $(J)[15,16]$. $J$ is defined as the maximum vertical distance between the ROC curve and the diagonal or chance line and is calculated as $J=\max$ imum \{sensitivity + specificity -1$\}[15,16]$. Chi-square tests and Wilcoxon rank sum tests were used to compare categorical and continuous variables, respectively, between the two groups. In analyses of related factors for $\mathrm{pN}+$, the clinical variables with $p<0.05$ in the univariate analysis were incorporated into the multivariate analysis to identify independent factors. All statistical analyses were performed using JMP 13 (SAS Institute, Cary, NC, USA), and $p<0.05$ was set as the level of significance.

\section{Results}

\section{Patient characteristics}

The clinical and pathological characteristics of the patients are shown in Table 1 . Patients were diagnosed with cT1 $(n=277)$ or cT2-T4 $(n=152)$, and pathologically diagnosed with pT1 $(n=277)$ or pT2-T4 $(n=152)$. Seventy-three patients (17.0\%) were diagnosed with $\mathrm{cN}+$ by conventional diagnostic modalities, and 116 patients (27.0\%) were pathologically diagnosed with $\mathrm{pN}+$. The incidence of $\mathrm{pN}+$ in $\mathrm{cT} 2-\mathrm{T} 4$ was $56.6 \%$, which was higher than that $(10.8 \%)$ in $\mathrm{cT} 1 \quad(p<0.001)$. The preoperative NLR ranged from 0.6 to 10.8 (median, 2.0). The NLR value for patients with CT2-T4 was $2.6 \pm 1.5$ (mean \pm standard deviation (SD)), which was higher than that $(2.2 \pm 1.2)$ for those with cT1 $(p=0.037)$.

\section{NLR according to $\mathrm{cN}$ and $\mathrm{pN}$}

The NLR value for patients with $\mathrm{cN}+$ or $\mathrm{cN} 0$ was $2.4 \pm$ 1.3 or $2.3 \pm 1.3$ (mean $\pm \mathrm{SD}$ ), respectively $(p=0.921)$. The NLR value for patients with $\mathrm{pN}+$ was $2.6 \pm 1.5$ (mean \pm $\mathrm{SD})$, which was higher than that $(2.2 \pm 1.2)$ for those with pN0 $(p=0.003)$. 
Table 1 Clinicopathological characteristics of the patients

\begin{tabular}{|c|c|c|c|c|}
\hline & cT1-T4 $(n=429)$ & cT1 $(n=277)$ & CT2-T4 $(n=152)$ & $P$ value \\
\hline \multicolumn{5}{|l|}{ Clinical characteristics } \\
\hline Sex, n (\%) & & & & 0.341 \\
\hline Male & $278(64.8)$ & $175(63.2)$ & $103(67.8)$ & \\
\hline Female & $151(35.2)$ & $102(36.8)$ & $49(32.2)$ & \\
\hline Age (years) & & & & 0.016 \\
\hline Median (range) & $67(29-89)$ & $66(35-89)$ & $69(29-89)$ & \\
\hline Mean \pm SD & $65.6 \pm 11.4$ & $64.7 \pm 11.2$ & $67.1 \pm 11.7$ & \\
\hline Tumor location, n (\%) & & & & $<0.001$ \\
\hline Upper & $108(25.2)$ & $67(24.2)$ & $41(27.0)$ & \\
\hline Middle & $204(47.6)$ & $154(55.6)$ & $50(32.9)$ & \\
\hline Lower & $117(27.3)$ & $56(20.2)$ & $61(40.1)$ & \\
\hline $\mathrm{cT}, \mathrm{n}(\%)$ & & & & - \\
\hline $\mathrm{T} 1$ & $277(64.6)$ & $277(100)$ & - & \\
\hline $\mathrm{T} 2$ & $116(27.0)$ & - & $116(76.3)$ & \\
\hline T3 & $34(7.9)$ & - & $34(22.4)$ & \\
\hline T4 & $2(0.5)$ & - & $2(1.3)$ & \\
\hline cN, n (\%) & & & & $<0.001$ \\
\hline NO (negative) & $356(83.0)$ & $267(96.4)$ & 89 (58.6) & \\
\hline $\mathrm{N}+$ (positive) & $73(17.0)$ & $10(3.6)$ & $63(41.4)$ & \\
\hline NLR & & & & 0.037 \\
\hline Median (range) & $2.0(0.6-10.8)$ & $2.0(0.6-10.8)$ & $2.1(0.6-10.5)$ & \\
\hline Mean \pm SD & $2.3 \pm 1.3$ & $2.2 \pm 1.2$ & $2.6 \pm 1.5$ & \\
\hline \multicolumn{5}{|l|}{ Pathological characteristics } \\
\hline pT, n (\%) & & & & $<0.001$ \\
\hline $\mathrm{T} 1$ & $277(64.6)$ & $245(88.4)$ & $32(21.1)$ & \\
\hline $\mathrm{T} 2$ & 49 (11.4) & $16(5.8)$ & $33(21.7)$ & \\
\hline T3 & $62(14.5)$ & $14(5.1)$ & 48 (31.6) & \\
\hline T4 & $41(9.6)$ & $2(0.7)$ & 39 (25.6) & \\
\hline pN, n (\%) & & & & $<0.001$ \\
\hline NO (negative) & $313(73.0)$ & $247(89.2)$ & $66(43.4)$ & \\
\hline $\mathrm{N}+$ (positive) & $116(27.0)$ & $30(10.8)$ & 86 (56.6) & \\
\hline
\end{tabular}

\section{ROC curve analysis}

The optimal cut-off value of NLR for predicting pN+ was 1.6 according to the ROC curve using the maximal Youden index (AUROC, 0.595; sensitivity, 83.6\%; specificity, 36.4\%) (Fig. 1). Thereafter, all subjects were divided into two groups as follows: the low NLR $($ NLR $<1.6)$ and high NLR (NLR $\geq 1.6)$ groups.

\section{Clinical factors associated with $\mathrm{pN}+$ in $\mathrm{GC}$}

The univariate and multivariate analyses of clinical factors associated with $\mathrm{pN}+$ in patients with EGC (cT1) or AGC (cT2-T4) are shown in Table 2. In patients with cT1, the univariate logistic analysis showed that a NLR $\geq 1.6$ was not significantly associated with $\mathrm{pN}+$ (odds ratio (OR) 2.253;
95\% confidence interval (CI) 0.942-6.266, $p=0.069$ ), and $\mathrm{cN}+$ was significantly correlated with $\mathrm{pN}+(\mathrm{OR}$ 9.680; 95\% CI 2.537-37.07, $p=0.002$ ). However, in patients with cT2$\mathrm{T} 4$, the univariate and multivariate logistic regression analysis identified NLR (OR 3.171; 95\% CI 1.448-7.235, $p=$ $0.004)$ and $\mathrm{cN}$ (OR 2.426; 95\% CI 1.221-4.958, $p=0.011)$ to be independently associated factors with $\mathrm{pN}+$.

\section{Clinical value of NLR as a predictor of $\mathrm{pN}+$ in advanced GC}

The diagnostic accuracy of NLR or conventional modalities for $\mathrm{pN}+$ in patients with AGC (cT2-T4) is shown in Table 3. The sensitivity, specificity, positive predictive value (PPV), negative predictive value 


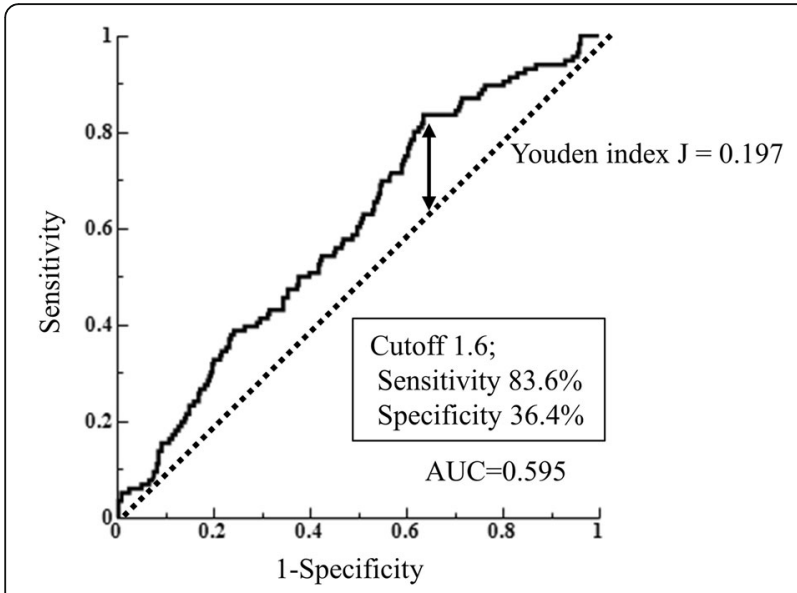

Fig. 1 ROC curve for NLR for predicting $p N+$ in patients with gastric cancer. ROC: receiver operating characteristic, NLR: neutrophil-tolymphocyte ratio, AUC: area under the curve

(NPV), and diagnostic accuracy of a NLR $\geq 1.6$ for $\mathrm{pN}+$ were $84.9,34.8,62.9,63.9$, and $63.2 \%$, respectively. Thus, the sensitivity was higher, specificity was lower, and diagnostic accuracy was slightly higher than those (50.0, 69.7, and 58.6\%, respectively) of conventional modalities. Next, the predictive ability of NLR for $\mathrm{pN}+$ was separately examined according to $\mathrm{cN}$ (N0 or $\mathrm{N}+$ ) (Table 4). When the subjects were limited to AGC patients with $\mathrm{cNO}$, the sensitivity and NPV of a $\mathrm{NLR} \geq 1.6$ for $\mathrm{pN}+$ increased further $(90.7$ and $81.0 \%$, respectively).

\section{Discussion}

Systemic inflammatory response plays an important role in cancer development and progression [6-8]. Therefore, the increase in NLR, due to the systemic inflammatory response induced by cancer, may be a novel diagnostic modality for $\mathrm{pN}+$ in GC. In the present study, the preoperative NLR was demonstrated to be independently associated with $\mathrm{pN}+$ in patients with AGC (cT2-T4), but not in those with EGC (cT1). In AGC, a NLR $\geq 1.6$ detected $\mathrm{pN}+$ with a higher sensitivity (84.9\%) than that (50.0\%) of the CT. The high sensitivity of the preoperative NLR suggests it to be a useful complementary modality in the assessment of $\mathrm{pN}+$ in AGC.

The therapeutic strategy for GC, including the extent of gastric resection and lymphadenectomy, is determined based on $\mathrm{CT}$ (T1 or T2-T4) and $\mathrm{cN}$ (N0 or $\mathrm{N}+$ ) [4]. However, it is challenging to accurately predict the pathological tumor stage, particularly $\mathrm{pN}$, because of the low diagnostic accuracy of conventional modalities $[5,12]$. In the present study, the sensitivity, specificity, and diagnostic accuracy of CT for detecting $\mathrm{pN}+$ (or $\mathrm{pN} 0)$ in patients with AGC were as low as 50.0, 69.7, and 58.6\%, respectively. On the other
Table 2 Clinical factors associated with $\mathrm{pN}+$ in patients with early or advanced gastric cancer

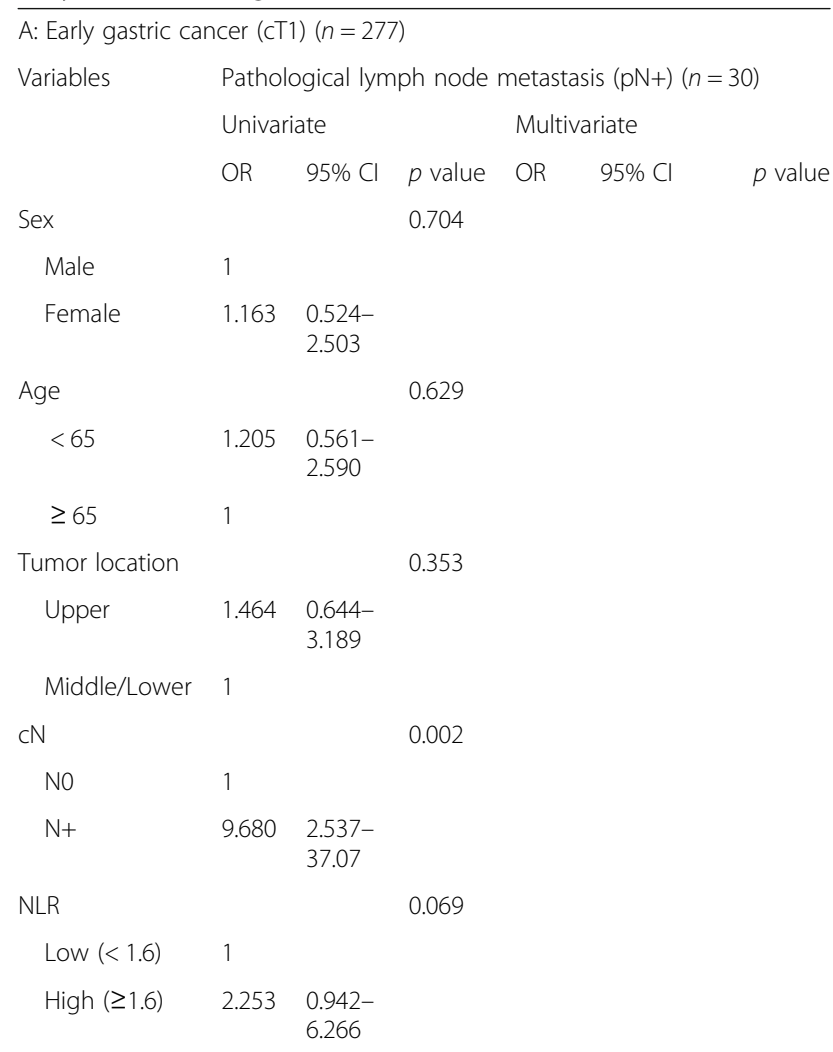

B: Advanced gastric cancer (cT2-T4) $(n=152)$

Variables Pathological lymph node metastasis $(\mathrm{pN}+)(n=86)$
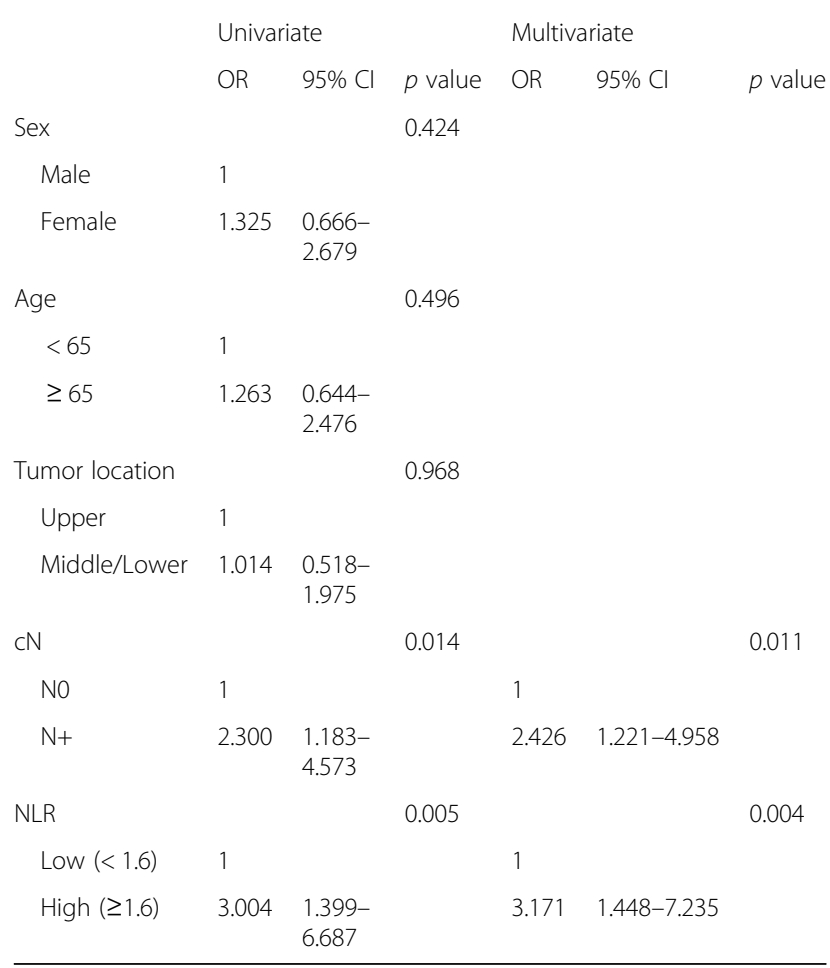

NLR neutrophil-to-lymphocyte ratio 
Table 3 Diagnostic accuracy for pN+ in patients with advanced gastric cancer

\begin{tabular}{|c|c|c|c|}
\hline \multicolumn{4}{|c|}{ A: Conventional modalities (CT) } \\
\hline & $\mathrm{pN}+$ & pNO & $n$ \\
\hline $\mathrm{cN}+$ & 43 & 20 & 63 \\
\hline cNO & 43 & 46 & 89 \\
\hline$n$ & 86 & 66 & 152 \\
\hline \multicolumn{4}{|l|}{ B: NLR } \\
\hline & $\mathrm{pN}+$ & $\mathrm{pNO}$ & $n$ \\
\hline High NLR ( $\geq 1.6)$ & 73 & 43 & 116 \\
\hline Low NLR $(<1.6)$ & 13 & 23 & 36 \\
\hline$n$ & 86 & 66 & 152 \\
\hline \multicolumn{4}{|c|}{ C: Diagnostic accuracy for pN+ } \\
\hline & $\mathrm{cN}+$ & High NLR ( $\geq 1.6)$ & \\
\hline Sensitivity & $50.0 \%$ (95\% Cl: $43.2-56.2)$ & $84.9 \%$ (95\% Cl: 78.9-90.1) & \\
\hline Specificity & $69.7 \%$ (95\% Cl: 60.8-77.8) & $34.8 \%$ (95\% Cl: 27.1-41.6) & \\
\hline Positive predictive value & $68.3 \%$ (95\% Cl: 58.9-76.7) & $62.9 \%$ (95\% Cl: 58.5-66.8) & \\
\hline Negative predictive value & $51.7 \%$ (95\% Cl: 45.1-57.7) & $63.9 \%$ (95\% Cl: 49.7-76.3) & \\
\hline Diagnostic accuracy & $58.6 \%$ (95\% Cl: 50.8-65.6) & $63.2 \%$ (95\% Cl: 56.4-69.0) & \\
\hline
\end{tabular}

NLR neutrophil-to-lymphocyte ratio

Table 4 Predictive ability of NLR for $\mathrm{pN}+$ separately examined according to $\mathrm{CN}$ in patients with advanced gastric cancer

\begin{tabular}{|c|c|c|c|}
\hline \multicolumn{4}{|l|}{$\mathrm{A}: \mathrm{CNO}$} \\
\hline & $\mathrm{pN}+$ & pNO & $n$ \\
\hline High NLR ( $\geq 1.6)$ & 39 & 29 & 68 \\
\hline Low NLR $(<1.6)$ & 4 & 17 & 21 \\
\hline$n$ & 43 & 46 & 89 \\
\hline \multicolumn{4}{|c|}{ Diagnostic accuracy for pN+ } \\
\hline & \multicolumn{3}{|c|}{ High NLR ( $\geq 1.6)$} \\
\hline Sensitivity & \multicolumn{3}{|c|}{90.7 \% (95\% Cl: 81.8-96.1) } \\
\hline Specificity & \multicolumn{3}{|c|}{37.0 \% (95\% Cl: 28.6-42.0) } \\
\hline Positive predictive value & \multicolumn{3}{|c|}{57.4 \% (95\% Cl: 51.7-60.8) } \\
\hline Negative predictive value & \multicolumn{3}{|c|}{81.0 \% (95\% Cl: 62.7-92.0) } \\
\hline \multicolumn{4}{|l|}{$\mathrm{B}: \mathrm{CN}+$} \\
\hline & $\mathrm{pN}+$ & pNO & $n$ \\
\hline High NLR ( $\geq 1.6)$ & 34 & 14 & 48 \\
\hline Low NLR $(<1.6)$ & 9 & 6 & 15 \\
\hline$n$ & 43 & 20 & 63 \\
\hline \multicolumn{4}{|l|}{ Diagnostic accuracy for pN+ } \\
\hline & \multicolumn{3}{|c|}{ High NLR ( $\geq 1.6)$} \\
\hline Sensitivity & \multicolumn{3}{|c|}{79.1 \% (95\% Cl: 72.6-86.3) } \\
\hline Specificity & \multicolumn{3}{|c|}{30.0 \% (95\% Cl: 16.0-45.5) } \\
\hline Positive predictive value & \multicolumn{3}{|c|}{70.8 \% (95\% Cl: 65.0-77.3) } \\
\hline Negative predictive value & \multicolumn{3}{|c|}{40.0 \% (95\% Cl: 21.3-60.7) } \\
\hline
\end{tabular}

NLR neutrophil-to-lymphocyte ratio hand, the diagnostic accuracy for detecting pT2-T4 (or pT1) was $85.1 \%$ (Additional file 1: Table S1), which was easier to diagnose than $\mathrm{pN}$. Positron emission tomography (PET) integrated with CT (PET-CT) may have played a role in the improved diagnostic accuracy for $\mathrm{pN}+$ by increasing the specificity [5]; however, the low sensitivity is one of the weak points of this modality. Therefore, the development of novel diagnostic tools is essential to increase the specificity and sensitivity for predicting $\mathrm{pN}+$.

Most previous studies focused on a high NLR as a useful predictor of long-term outcomes in patients with GC $[10,17,18]$. Indeed, in the targeted cohorts of the present study, the postoperative 5-year overall and cancer-specific survival rates of patients with a NLR $\geq$ 1.6 were significantly poorer than those with a NLR $<$ 1.6 (data not shown). However, few studies have assessed the clinical significance of NLR as a diagnostic tool for $\mathrm{pN}+$ in GC. Shimada et al. reported that the mean preoperative NLR in GC patients with $\mathrm{pN}+$ was 2.91 , which was significantly higher than that (2.40) in patients with pNO [10], but the predictive ability of NLR for pN+ was not examined in detail. Zhang et al. estimated an optimal cutoff value of NLR of 2.0 (sensitivity, 52.6; specificity, 54.4; AUROC, 0.594), and a $\mathrm{NLR} \geq 2.0$ was significantly associated with $\mathrm{pN}+$ by univariate analysis [18]; however, they neither assessed the influence of potential confounding factors, such as $\mathrm{cT}$ and $\mathrm{cN}$, nor compared the diagnostic ability with conventional diagnostic modalities. 
The present study was the first to explore whether the preoperative NLR is a predictor for $\mathrm{pN}+$ independent of $\mathrm{cN}+$, and to examine the diagnostic accuracy of NLR for $\mathrm{pN}+$ in comparison with conventional modalities. As a result, a NLR $\geq 1.6$ was found to be an independent predictor for $\mathrm{pN}+$ in AGC (cT2-T4) with a slightly higher OR than conventional diagnostic modalities. In EGC (cT1), however, cN+ was not significantly correlated with $\mathrm{pN}+$. Although the small sample size may be one of the responsible factors for the negative result in CT1, our study suggested that NLR was not superior to conventional modalities for predicting $\mathrm{pN}+$ among patients with cT1. The correlation between the NLR value and cT may also have affected the results; however, the cut-off values of NLR for predicting $\mathrm{pN}+$ determined by the ROC curve were 1.7 for CT1 and 1.6 for cT2-T4 (data not shown), which were both similar to the value for cT1-T4. Meanwhile, a NLR $\geq 1.6$ may also be a good predictor of pT2/T3/T4 (vs pT1); however, the diagnostic accuracy (48.3\%) was lower than that (85.1\%) of conventional modalities such as upper endoscopy, barium meal examination and abdominal CT (Additional file 1: Table S1).

Although it has the potential to predict $\mathrm{pN}+$ independent of $\mathrm{cN}+$, the diagnostic accuracy of a NLR $\geq 1.6$ for $\mathrm{pN}+$ in AGC was only $63.2 \%$, which was comparable to that of $\mathrm{cN}+$. Actually, in the McNemar test, there was not a significant difference in the diagnostic accuracy for $\mathrm{pN}+$ between a NLR $\geq 1.6$ and $\mathrm{cN}+(p=0.175)$ (data not shown). As the low specificity was the major reason for the poor diagnostic accuracy, attention should be paid to the high incidence of false-positive cases when using NLR to predict $\mathrm{pN}+$. On the other hand, the high sensitivity of NLR for $\mathrm{pN}+$ was of note, i.e., a NLR $<1.6$ may aid in the specific diagnosis of $\mathrm{pN}+$. To clarify the most effective clinical use of NLR in combination with conventional diagnostic modalities, the predictive ability of NLR for $\mathrm{pN}+$ was separately examined according to $\mathrm{cN}$. When the subjects were limited to AGC patients with cN0, the sensitivity and NPV of a NLR $\geq 1.6$ for $\mathrm{pN}+$ increased further (90.7 and $81.0 \%$, respectively). Therefore, a NLR $<1.6$ may aid in the prediction of pN0, especially in combination with conventional diagnostic modalities.

The present study had several limitations that should be considered. First, the retrospective and single-center nature of the study may have generated selection bias in the cohort, and the number of study patients was relatively small, which may have limited the statistical power. Second, as the cut-off value of NLR was calculated only by a mathematical method, the low specificity of a NLR $\geq 1.6$ for $\mathrm{pN}+$ is a problem to be solved. Third, unfortunately, we could not find a correlation between preoperative NLR value and the number of pathological positive lymph nodes (Spearman's rank correlation coefficient $(\rho)$ : $0.1224, p=0.011$ ) (data not shown); thus, it remains unclear whether biologically NLR is driving lymph node metastasis. Fourth, this study examined only NLR, but other immune-nutritional markers such as prognostic nutritional index (PNI) may also be useful predictors of $\mathrm{pN}+$. However, to the best of our knowledge, this is the first report to present the novel potential of preoperative NLR for predicting $\mathrm{pN}$ in patients with AGC in comparison with conventional modalities. As the number of elderly patients with GC who have many comorbid diseases and poor organ function is increasing [19-22], limited lymph node dissection may be reasonable for such patients when pNO is highly suspected based on the combined use of conventional modalities and NLR. The results of the present study and the optimal cut-off value of NLR need to be validated in further studies with large sample sizes to develop the sophisticated treatment strategies based on the reliable $\mathrm{cN}$.

\section{Conclusion}

The preoperative NLR may be a useful complementary diagnostic tool in the assessment of $\mathrm{pN}+$ in AGC because of its higher sensitivity and NPV than conventional diagnostic modalities.

\section{Supplementary information}

Supplementary information accompanies this paper at https://doi.org/10. 1186/s12885-019-6404-8.

Additional file 1: Table S1. Diagnostic accuracy for $\mathrm{pT} 2 / \mathrm{T} 3 / \mathrm{T} 4$ in patients with gastric cancer.

\section{Abbreviations}

AGC: Advanced gastric cancer; AUROC: Area under the receiver operating characteristic curve; CBC: Cell blood count; CECT: Contrast-enhanced computed tomography; Cl: Confidence interval; CT: Computed tomography; EGC: Early gastric cancer; EUS: Endoscopic ultrasound; GC: Gastric cancer; JCGC: Japanese classification of gastric carcinoma; JGCTG: Japanese gastric cancer treatment guidelines; KPUM: Kyoto Prefectural University of Medicine; NAC: Neoadjuvant chemotherapy; NLR: Neutrophil-to-lymphocyte ratio; NPV: Negative predictive value; OR: Odds ratio; PET: Positron emission tomography; PET-CT: Positron emission tomography integrated with computed tomography; PNI: Prognostic nutritional index; PPV: Positive predictive value; ROC: Receiver operating characteristic; SD: Standard deviation; WBC: White blood cell count

\section{Acknowledgements}

No acknowledgements.

\section{Authors' contributions}

TK1 contributed to the study conception and design. TK1 and TK2 acquired data. TK1, TK2, TK3, KS, HK, AS, KO, HF, MK, TA, RM, YM, YK, HI and MN contributed to the analysis and interpretation of data. TK1 and TK2 wrote the manuscript. EO made the critical revision. All authors have read and approved the manuscript.

\section{Funding}

No funding.

Availability of data and materials

The datasets used and/or analyzed during the current study are available from the corresponding author on reasonable request. 


\section{Ethics approval and consent to participate}

All procedures performed in studies involving human participants were in accordance with the ethical standards of the institutional and/or national research committee and with the 1964 Helsinki declaration and its later amendments or comparable ethical standards. This retrospective study was approved by the Medical Ethics Review Committee of the Kyoto Prefectural University of Medicine (Approved No. ERB-C-1373). It was determined to be a retrospective analysis of de-identified data, and written informed consent was waived for the individual participants included in the study in accordance with the standards of the Kyoto Prefectural University of Medicine Institutional Medical Ethics Review Committee.

\section{Consent for publication}

Not applicable.

\section{Competing interests}

The authors declare that they have no competing interests.

Received: 28 August 2019 Accepted: 26 November 2019

Published online: 05 December 2019

\section{References}

1. Bray F, Ferlay J, Soerjomataram I, Siegel RL, Torre LA, Jemal A. Global cancer statistics 2018: GLOBOCAN estimates of incidence and mortality worldwide for 36 cancers in 185 countries. CA Cancer J Clin. 2018 Nov;68(6):394-424.

2. Komatsu S, Ichikawa D, Miyamae M, Kosuga T, Okamoto K, Arita T, et al. Positive lymph node ratio as an Indicator of prognosis and local tumor clearance in N3 gastric Cancer. J Gastrointest Surg. 2016 Sep;20(9):1565-71.

3. Songun I, Putter H, Kranenbarg EM, Sasako M, van de Velde CJ. Surgical treatment of gastric cancer: 15-year follow-up results of the randomised nationwide Dutch D1D2 trial. Lancet Oncol. 2010 May;11(5):439-49.

4. Japanese Gastric Cancer Association. Japanese gastric cancer treatment guidelines 2014 (ver. 4). Gastric Cancer. 2017;20(1):1-19.

5. Kudou M, Kosuga T, Kubota T, Okamoto K, Komatsu S, Shoda K, et al. Value of preoperative PET-CT in the prediction of pathological stage of gastric Cancer. Ann Surg Oncol. 2018 Jun;25(6):1633-9.

6. Hanahan D, Weinberg RA. Hallmarks of cancer: the next generation. Cell. 2011 Mar 4;144(5):646-74.

7. Mantovani A, Allavena P, Sica A, Balkwill F. Cancer-related inflammation. Nature. 2008 Jul 24;454(7203):436-44.

8. Zahorec R. Ratio of neutrophil to lymphocyte counts--rapid and simple parameter of systemic inflammation and stress in critically ill. Bratisl Lek Listy. 2001;102(1):5-14.

9. Kubo H, Murayama Y, Arita T, Kuriu Y, Nakanishi M, Otsuji E. The prognostic value of preoperative neutrophil-to-lymphocyte ratio in colorectal Cancer. World J Surg. 2016 Nov;40(11):2796-802.

10. Shimada H, Takiguchi N, Kainuma O, Soda H, Ikeda A, Cho A, et al. High preoperative neutrophil-lymphocyte ratio predicts poor survival in patients with gastric cancer. Gastric Cancer. 2010 Aug;13(3):170-6.

11. Sierzega M, Lenart M, Rutkowska M, Surman M, Mytar B, Matyja A, et al. Preoperative neutrophil-lymphocyte and lymphocyte-monocyte ratios reflect immune cell population rearrangement in Resectable pancreatic Cancer. Ann Surg Oncol. 2017 Mar;24(3):808-15.

12. Hasegawa S, Yoshikawa T, Shirai J, Fujikawa H, Cho H, Doiuchi T, et al. A prospective validation study to diagnose serosal invasion and nodal metastases of gastric cancer by multidetector-row CT. Ann Surg Oncol. 2013 Jun;20(6):2016-22.

13. Yang DM, Kim HC, Jin W, Ryu CW, Kang JH, Park CH, et al. 64 multidetectorrow computed tomography for preoperative evaluation of gastric cancer: histological correlation. J Comput Assist Tomogr. 2007 Jan-Feb;31 (1):98-103.

14. Japanese Gastric Cancer Association. Japanese classification of gastric carcinoma: 3rd English edition. Gastric Cancer. 2011;14(2):101-12.

15. Fluss $R$, Faraggi $D$, Reiser B. Estimation of the Youden index and its associated cutoff point. Biom J. 2005 Aug;47(4):458-72.

16. Perkins NJ, Schisterman EF. The inconsistency of "optimal" cutpoints obtained using two criteria based on the receiver operating characteristic curve. Am J Epidemiol. 2006 Apr 1;163(7):670-5.

17. Saito H, Kono Y, Murakami Y, Shishido Y, Kuroda H, Matsunaga T, et al. Prognostic significance of the preoperative ratio of $\mathrm{C}$-reactive protein to albumin and neutrophil-lymphocyte ratio in gastric Cancer patients. World J Surg. 2018 Jun;42(6):1819-25.
18. Zhang LX, Wei ZJ, Xu AM, Zang JH. Can the neutrophil-lymphocyte ratio and platelet-lymphocyte ratio be beneficial in predicting lymph node metastasis and promising prognostic markers of gastric cancer patients? Tumor maker retrospective study. Int J Surg. 2018 Aug;56:320-7.

19. Kosuga T, Ichikawa D, Okamoto K, Komatsu S, Konishi H, Takeshita H, et al. Impact of age on early surgical outcomes of laparoscopy-assisted gastrectomy with suprapancreatic nodal dissection for clinical stage I gastric cancer. Anticancer Res. 2015 Apr;35(4):2191-8.

20. Ruspi L, Galli F, Pappalardo V, Inversini D, Martignoni F, Boni L, et al. Lymphadenectomy in elderly/high risk patients: should it be different? Transl Gastroenterol Hepatol. 2017;2:5.

21. Takeshita H, Ichikawa D, Komatsu S, Kubota T, Okamoto K, Shiozaki A, et al. Surgical outcomes of gastrectomy for elderly patients with gastric cancer. World J Surg. 2013 Dec;37(12):2891-8.

22. Yang JY, Lee HJ, Kim TH, Huh YJ, Son YG, Park JH, et al. Short- and longterm outcomes after Gastrectomy in elderly gastric Cancer patients. Ann Surg Oncol. 2017 Feb;24(2):469-77.

\section{Publisher's Note}

Springer Nature remains neutral with regard to jurisdictional claims in published maps and institutional affiliations.

\section{Ready to submit your research? Choose BMC and benefit from:}

- fast, convenient online submission

- thorough peer review by experienced researchers in your field

- rapid publication on acceptance

- support for research data, including large and complex data types

- gold Open Access which fosters wider collaboration and increased citations

- maximum visibility for your research: over $100 \mathrm{M}$ website views per year

At BMC, research is always in progress.

Learn more biomedcentral.com/submissions 